APA: León Molina, J. E. (2020). Calificación lógico-moral de los derechos humanos. En O. A. Agudelo Giraldo (Ed.), Derechos humanos, democracia y poder judicial (págs. 43-55). Editorial Universidad Católica de Colombia. https://doi.org/10.14718/9789585133020.2020.2

Chicago: León Molina, Jorge Enrique, “Calificación lógico-moral de los derechos humanos.” En Derechos humanos, democracia y poder judicial, editado por Oscar Alexis Agudelo Giraldo, 43-55. Bogotá:

Editorial Universidad Católica de Colombia, 2020. doi: 10.14718/9789585133020.2020.2

\title{
CALIFICACIÓN LÓGICO-MORAL DE LOS DERECHOS HUMANOS
}

Jorge Enrique León Molina*

\section{Introducción}

El objeto de investigación del presente artículo se recoge en la siguiente pregunta: ¿Cómo se califican normativa y lógicamente los derechos humanos desde su órbita moral? De entrada, este interrogante pone a quien lo lee en serios apuros, en cuanto la adecuación normativa de principios inherentes a los sujetos podría inducir a modelos naturalistas que, de muchas formas positivas, se han visto como algo anacrónico.

Sin embargo, desde el Derecho Natural solo se enuncian como derechos, con una amplitud de posibilidades bastante problemática, mientras que el positivismo plantea numerosas dificultades para su axiomatización. Esto último, debido a que su genericidad afecta el estatus científico del Derecho, en la medida en que no tiene posibilidad de reducción y demostración.

La determinación de "normas morales", en el modo de un sistema positivo, supone el hecho de comprender el alcance y la delimitación de esas reglas como,

\footnotetext{
* Abogado de la Universidad Católica de Colombia y Magister en Filosofía del Derecho de la Universidad Libre de Colombia. Docente-investigador de la facultad de Derecho de la Universidad Católica de Colombia. Coautor de libros como Teoría jurídica y enseñanza del Derecho. E-mail: jeleon@ucatolica.edu.co. Bogotá D.C., Colombia.
} 
a lo sumo, de tipo operativo en el marco de la lógica, lo cual se toma en consideración a partir de los llamados “permisos débiles". Además, esto determina cómo la lógica jurídica no es suficiente para abarcar criterios de reglas de corte moral.

Pero, ¿cómo poder categorizar las normas morales? El presente escrito tiene en cuenta tanto las nociones de Dworkin como las de Bayón, en virtud de las cuales se hacen inclusiones de criterios morales en forma contingente, al más puro estilo del positivismo incluyente. En este, la nominación de principios y valores ontológicamente diferentes al clásico estatuto positivista, enriquece el debate sobre la vinculación de reglas morales como derechos humanos en el ordenamiento jurídico.

\section{Derechos humanos: peculiaridades iusfilosóficas}

La noción de "derechos humanos" es problemática en el estudio del Derecho, dado que aunque tenga una clara aceptación desde el positivismo, también tiene una dimensión moral que vicia su interpretación en el caso concreto. Sin embargo, el reconocimiento de ese tipo de derechos "adquiere una importancia radical para cuestionar leyes, instituciones, medidas o acciones" (Nino, 1989, p. 14). Es decir, son prerrogativas que sirven como oposición a la visión absolutista del imperio del Derecho.

Esto implica que los derechos humanos no solo se circunscriben a su consagración positivista, en otras palabras, a su adecuación en la forma de circunstancias puramente normativas, sino que además constituyen un conjunto de normas oponibles a sistemas jurídicos, que pretenden garantizar mínimos básicos de dignidad en la existencia humana. Así, Pico Della Mirandola (2010) expone:

A ti hombre, no te he dado ni un lugar determinado, ni un aspecto propio, ni una prerrogativa peculiar, con el fin de que poseas el lugar, el espacio, el aspecto y la prerrogativa que conscientemente elijas y que de acuerdo con tu intención obtengas y conserves (p. 4).

Para Nino, los derechos humanos surgen "no de un sistema normativo basado en actos contingentes, sino en la justificación que se hace de estos en el seno del sistema normativo" (Nino, 1989, p. 15). Lo anterior implica que dichas expresiones son la condición básica que surge al interior del modelo normativo, para 
salvaguardar las garantías o principios mínimos a los que tiene derecho el ser humano, en el marco de todo el sistema jurídico.

En este punto, la justificación interna es problemática, dado que puede surgir de múltiples acciones: del desarrollo espiritual de la humanidad, de conquistas de la modernidad, de acuerdos sociales, de procesos revolucionarios o de procesos que obedecen a necesidades sociales, políticas o jurídicas. Estas podrían incluso ser exógenas al sistema jurídico, pero afectarlo directamente, dado que "lo que persiguen los individuos es ir en contra de comportamientos que sofoquen la variedad natural de sus carácteres, en pro del bienestar y la libertad" (Bobbio, 1993, p. 27).

Por lo tanto, Nino plantea una raíz naturalista de la justificación de los derechos humanos, determinada en una forma moral de razonamiento. Para ello, presenta dos tesis fundamentales:

1. El origen de los principios básicos de la existencia humana no está fundamentado en las instituciones jurídicas, sino en la universalidad de las virtudes personales de todos los individuos, expresas en forma de "libertades como restricciones a la libertad real del ser humano" (Von Wright, 2002, p. 62). De esta manera se procura, a lo sumo, un criterio básico de justicia.

2. El derecho se justifica si y solo si procura una búsqueda de criterios de justicia social.

Por otro lado, en el positivismo no es posible la determinación de principios universalmente válidos, sin que estos puedan llegar a ser cognoscibles por medio de procedimientos racionales. Por esta razón, es necesario analizar el concepto “derechos humanos" desde su dimensión semántica, lo que debe hacerse más allá de una distinción ontológica, en virtud de la cual "la existencia de un concepto no atañe al conocimiento que se tenga de él" (Nino, 1989, p. 19).

Lo anterior, con el fin de no afectar tal reconocimiento con la subjetividad que traería consigo la interpretación moral de los principios propios del Derecho Natural, ya que "al hacer categorías basadas en derechos demasiado genéricos, es imposible su fundamentación racional; por eso son llamados derechos humanos" (Garzón, 1997, p. 410). 
Sin embargo, para una mejor comprensión de la órbita moral de dichos derechos, Nino expone ciertas características que, a lo sumo, permiten una interpretación desde el positivismo de tales principios y de su naturaleza:

1. Son principios morales que están supeditados a la determinación de su existencia, a través de su validez y aceptabilidad por el ordenamiento jurídico. En otras palabras, son principios dados por una moral, que tiene su incidencia y origen desde el Derecho Positivo. En este "se enuncian las normas básicas sin las cuales es imposible lograr una base social estructurada, o sin las cuales una sociedad ordenada se encamina a ciertos fines específicos en pro tanto del bienestar como del equilibrio social" (Fuller, 1967, p. 13).

2. La aceptación de tales principios es la justificación final de las conductas que describe. Esto significa que son la última herramienta del sistema positivo para delimitar el alcance y la validez fáctica de un principio moral.

3. Esta interpretación desde la moral positiva sirve como modelo para cualquier conducta, ya sea desde el agente, desde "la acción o desde quien sufre/siente la acción" (Nino, 1989, p. 20). Sin embargo, su examen no corresponde a un juicio a priori sobre su adecuación a la acción, sino que, respecto a esta, se hace un examen básico que tiene en cuenta el contenido del principio empleado, de manera similar al modo lógico de la subsunción.

Estas tres interpretaciones posibilitan la inclusión de criterios morales a un análisis positivo, mediante el juicio de adecuación basado en elementos de validez, legitimidad y pertinencia de dichos criterios al sistema jurídico. Tal juicio sirve no solo como un mecanismo de axiomatización, sino también como un determinante de su implicación positiva, dado que "por ley moral se deben entender las indispensables normas morales puestas en defensa ya del individuo como de la comunidad" (Haberle, 2003, p. 61).

A la luz del Derecho Positivo, del análisis de la naturaleza moral de los derechos humanos, se podría extraer su carácter problemático, cometiendo el error de no considerarlos como criterios válidos de justicia, en cuanto no tienen un asiento 
en el sistema positivista. Esta circunstancia afecta tanto su eficacia y su obediencia, así como su aplicabilidad. Sin embargo, para Nino (1989)

la identificación de un sistema jurídico es una cuestión teórica que puede resolverse en forma valorativamente neutra; la decisión acerca de qué actitudes y qué curso de acción debe tomarse frente a un cierto orden jurídico, es una cuestión práctica que no puede resolverse sin comprometerse, explícita o implícitamente, con ciertos criterios de justicia y moralidad social (p. 21).

Esto implica que para el estudio de los hechos relevantes, el modelo del positivismo supedita su valoración a unas condiciones de inclusión al sistema jurídico, por medio de proposiciones surgidas de la adecuación de esos hechos a normas jurídicas propias del Derecho. Dichas normas, al ser producto de la actividad judicial, generan la inclusión de esos conceptos morales ya positivizados.

Dicha posibilidad es contemplada por Kelsen, quien, siguiendo a Smend, dice que "una de las funciones del Estado es integrar factores que agrupan, en sí mismos, como sistema, la realidad estatal" (Kelsen, 2009, p. 105). Respecto a la cita, hay que colegir que hoy en día parte de la realidad jurídica estatal está inmersa en la posibilidad de abarcar circunstancias fácticas más allá del ordenamiento positivo vigente.

\section{Naturalismo, positivismo y derechos humanos}

La adecuación de hechos a la luz del Derecho tiene dos posturas de estudio, oponibles desde antaño: el naturalismo y el positivismo. Por un lado, en el marco del naturalismo, a partir de principios universales de justicia, se establecen las proposiciones de interpretación de derechos, generando un amplio margen de indeterminación sobre el sentido y significación de los mismos, amplificando, hasta el punto de la vaguedad, las consideraciones valorativas de los principios.

Por otro lado, el marco del positivismo también supone un yerro jurídico, al plantear que las soluciones judiciales de contextos de derechos humanos obedecen a criterios de adecuación de cuestiones normativas. De hecho, no es posible hacer un concepto puramente descriptivo de la validez e implicación de un derecho humano en un contexto determinado. Así, los asuntos interpretativos del Derecho Positivo solo aportan a la adecuación cerrada de hechos puntuales, lo que, en palabras de Nino, supone que "tales proposiciones describirían, en última 
instancia, una secuencia compleja de hechos que, como tales, son compatibles con cualquier tipo de decisión" (Nino, 1989, p. 21).

Sin embargo, a tenor de lo expuesto, no debe pasarse por alto que las decisiones jurídicas respecto a la aplicación, validez o determinación de los derechos humanos están supeditadas a su calificación moral, circunstancia que excede toda calificación positiva. Lo anterior, dado que:

1. No siempre hay una norma jurídica para el caso que se somete a estudio, es decir, la justificación jurídica del mismo es producto de un análisis moral muchas veces incompleto.

2. En caso de que sea posible su axiomatización positiva, la justificación judicial está viciada por criterios valorativos, dado que la norma, desde su formulación, trae elementos de textura abierta que posibilitan la amplificación de su interpretación hacia estados más allá del normativo.

Esto permite concluir, en palabras de Nino, que aunque las normas jurídicas se resuelven en juicios de "adhesión normativa" (Nino, 1989, p. 23), lo que se hace es interpretar tanto la voluntad de quien las emite, así como del o de los juicios valorativos que están implícitos en la misma, los cuales amplifican su espectro normativo. Eso pasa cuando se interpretan principios o valores, tales como los derechos humanos.

Entonces, un examen de positivismo incluyente permite la comprensión de criterios de justicia y moralidad social (Nino, 1989), en el que se puede hacer la adecuación sistémica de elementos propios de moralidades positivistas, así como un análisis de circunstancias fácticas y sus implicaciones en diversas formas del razonamiento jurídico.

Esto se da incluso si el derecho humano está por encima del derecho nacional, caso en el que los procedimientos morales se hacen más relevantes para interpretar razones de la observancia de principios válidos. Dicha coincidencia se hace por vía interpretativa, llevando su aplicación más allá del ordenamiento positivo vigente y justificándose en la decisión judicial que la posibilita. Este ejercicio es un asunto constante, dado que el reconocimiento jurídico de los derechos humanos, ya sea desde la órbita nacional o desde la internacional, solo procura mostrar los medios para garantizar su no violación. 


\section{Anotaciones para la calificación lógico-jurídica de los derechos humanos}

Los derechos humanos, desde su dimensión originaria -de corte moral-, presentan un problema fundamental en cuanto a su identificacion lógico-jurídica, que tiene que ver con su formulación como una prerrogativa que permita, prohiba u obligue la realización de acciones en el marco de los derechos subjetivos, al margen de un sistema jurídico común.

Desde la determinación normativa de las conductas, Von Wright enuncia tres operadores que las describen. Por un lado, está la obligación, en virtud de la cual "un estado de cosas se debe hacer de forma ilimitada y sin posibilidad alguna de su no ejecución; esto implica que su uso prescriptivo determina la realización irrestricta de su condición (Von Wright, 1997, p. 11). Por otro lado, se tiene que la permisión radica en la posibilidad de realización, o no, de una acción determinada, en la cual "se permite el estado de cosas que resulta tras el actuar del agente" (Echave, Urquijo \& Guibourg, 2008, p. 121). Por último, una prohibición se enuncia como el caso en que una conducta no puede ser en ninguna medida posible, so pena de infringir el sistema del Derecho.

Se tiene que interpretar si tal derecho es, en palabras de Nino, uno básico o uno derivado (Nino, 1989), es decir, si garantiza solo limitaciones o prescripciones de la libertad, o si, por el contrario, permite o prohibe acciones determinadas. A continuación se mostrará cómo esta categorización se puede adecuar al discurso normativo.

Derechos humanos en la forma de reglas sin prohibición. En esta primera categoría, las normas que cobijan la interpretación de los derechos humanos no contienen una prohibición de forma directa. Estas normas, a lo sumo, abarcan un criterio de permisión que funciona como "una norma de clausura que permit[e] una conducta no prohibitiva" (Nino, 1989, p. 25).

Sin embargo, Agudelo define el permiso débil como "aquel que implica la valoración de aquellas circunstancias no reguladas por el Derecho" (Agudelo, 2017, p. 150). De tal modo que, desde esta categoría, se puede inferir que al tener un derecho, se evidencia la falta de calificación de la acción que se presume que regula. Es decir, se expresa como un deber vacío, en donde no se dice absolutamente nada de cómo se debe reaccionar respecto a la ejecución o no de la acción que se pretende realizar, ni tampoco sobre algún tipo de protección sobre la misma. 
Esta falta de calificación es similar a la noción de laguna axiológica, la cual expresa que no se tiene completamente un vacío en la interpretación de la norma, sino en "la calificación de las hipótesis de relevancia de la solución del caso concreto, en relevancia con el sistema jurídico" (Alchourrón \& Bulygin, 2013, p. 156).

Lo anterior implica que las lagunas axiológicas traen consigo la determinación de propiedades relevantes para la interpretación y solución del caso concreto, partiendo de un conjunto de elementos morales que permiten la adecuación de la respuesta más acorde con la facticidad de los elementos en controversia jurídica. De ahí se colige que "la pertinencia de la existencia de la laguna axiológica se posibilita en la existencia de defectos, ya sean políticos, morales, jurídicos o sociales que le den origen" (Alchourrón \& Bulygin, 2013, p. 157).

Derechos humanos en la forma de reglas de permisión. Existen normas que otorgan la facultad de realización de una acción, en las cuales se enuncia una conducta de hacer, pero es discutible si estas tienen inserta, además, una forma de deber. Para ello, "es necesaria la determinación pragmática de la formulación normativa descrita, con el fin de determinar cuál es el fin que se persigue en el destinatario con esa norma formulada" (Nino, 1989, p. 26).

Como los derechos humanos surgen de prerrogativas morales demasiado genéricas, el límite de una permisión está dado por la determinación de otra. En este caso, se debe establecer el mínimo espectro de cumplimiento de las permisiones, a fin de no superponer una a la otra, puesto que, de esta manera, en el curso de su cumplimiento podrían hacerse contradictorias entre sí.

Aunque para Von Wright las normas morales no tienen la misma categoría que las jurídicas, lo cierto es que estos dos tipos presentan elementos comunes que las sitúan como normas, en virtud de "su consideración teleológica, su vocación prescriptiva y su vocación, ya sea obligatoria, permitiva o prohibitiva en pro de mantener o procurar una correcta acción en procura del bienestar y la felicidad" (Von Wright, 1979, p. 31).

Derechos humanos como obligaciones correlativas. Esta es la forma básica de toda formulación normativa, en virtud de la cual se impone una determinada obligación, un deber positivo en relación a una conducta particular. Las obligaciones se pueden generar en campos tan específicos como los derechos subjetivos 
o en campos tan amplios como "aquellos [derechos] que constituyen un perímetro protector para el ejercicio de las libertades" (Nino, 1989, p. 27).

De todo esto se puede colegir que la estructura propia de los derechos humanos, al ser genéricos y morales, necesariamente va más allá de la lógica de la determinación de los deberes, razón por la que la construcción de los espectros morales tiene una dinámica diferente.

\section{Calificación moral de los derechos humanos}

El punto de partida para realizar el análisis de la calificación moral de un tipo de norma específica es el principio de moralidad que describe Ávila, en virtud del cual se establecen valores fundamentales, que implican "el deber de tenerlos en consideración en el ejercicio de la actividad administrativa" (Ávila, 2011, p. 84). En esta se determina que la primacía de los actos que otorgan o determinan la axiomatización de principios morales no sea fruto de una actividad arbitraria de un sujeto, sino una prerrogativa estructurada en la función del sistema jurídico.

La función del principio de moralidad es plantear una actitud del sistema normativo-político para el tratamiento de normas específicas, aunque dicha interpretación no esté contenida en el ordenamiento jurídico. Como expone Ávila (2011),

este examen se hace para determinar la racionalidad de toda interpretación de formulaciones morales a la luz de la función normativa, promoviendo la moralidad en la función de la administración de justicia que les permita su incorporación y el castigo coactivo de su incumplimiento (p. 86).

Esto lleva a pensar que del principio de moralidad surgen los derechos correlativos, que vinculan elementos más allá de los contenidos en todo el sistema jurídico, e incluso más allá del sistema político.

Hablar de derechos correlativos supone, a lo sumo, el establecimiento de obligaciones, permisiones o prohibiciones que estén íntimamente relacionadas entre sí, que sean determinables las unas a las otras y que tengan un nivel de deber similar entre ellas. Esto solo corrobora la facultad del derecho de construirse a sí mismo, en variables sistémicas que procuren una fundamentación positiva. Sin embargo, Nino expone que el caso de los principios generales supone "una concepción moral cuyos principios básicos establezcan tanto deberes, como derechos y fines sociales" (Nino, 1989, p. 32). 
La función de los principios, en casos concretos, obedece a una lógica gradual que se realimenta en cada uno de ellos, permitiendo la aplicación de reglas de axiomatización que resuelven los conflictos entre normas morales, en virtud de la función jurisdiccional del Derecho.

Siguiendo a Dworkin, Pérez Bermejo entiende que el modelo normativo es "frecuentemente un compromiso de principios y valores" (Pérez Bermejo, 2010, p. 99), en el que las reglas primarias, desde un ámbito meramente legal, determinan las dimensiones de peso de cada principio como regla moral ulterior. De esta manera, constituyen "las diferentes formas de comportamiento y relación de los principios, sea en el sistema o en el caso concreto" (Pérez Bermejo, 2010, p. 102).

Así, se puede afirmar que los principios, como reglas morales supranormativas, dotan de validez y sentido las reglas primarias emitidas por el sistema jurídico, en caso de garantías generales de actos morales internos de los hombres.

Por otro lado, Dworkin afirma que los principios pueden surgir espontáneamente en la regulación jurídica de espectro moral, a partir de la función judicial. Esto los amplifica a tal punto que ya no son susceptibles de enunciación taxativa en una lista cerrada. Dicho elemento se determina porque

los Principios escapan de toda formulación axiomática que permita su deducción mediante algún tipo de algoritmo; dado que, por su naturaleza compleja, dotaría al sistema de imprecisiones a la hora de reconstruir el sistema jurídico. Generando problemas a la hora de deducir respuestas a problemas jurídicos (Dworkin, 2012, p. 197).

Así, en medio de la distinción normas-principios, los conflictos entre estas entidades que se consideran de diferente valor, son difíciles de solucionar incluso para la lógica tradicional. Por esta razón, el mismo Dworkin critica el modelo piramidal del Derecho, junto con la denominada Jurisprudencia Mecánica, que hace referencia a que la solución a un caso concreto "obedece a un algoritmo lógico que subsume un hecho en una determinada formulación normativa" (Dworkin, 2012, p. 43).

Siguiendo lo anterior, Dworkin enfrenta la vocación moral de los principios con las bases axiomático-jurídicas del sistema Jurídico-Positivista, demostrando en esta tensión la imposibilidad de completitud de un sistema de este tipo, dado que es prácticamente improbable determinar la totalidad de sus principios. Sin embargo, 
una tesis así permite explicar, en términos estrictamente realistas, la organización jerárquica del orden jurídico: todo se reduce a ideas, a mitos más o menos útiles, apoyados en la actitud general de las personas, y que colocan a ciertas normas en posiciones claves que les permiten presionar sobre los ciudadanos (Guibourg, 2010, p. 70).

A partir de esto, Bayón especifica que las reglas morales están "caracterizadas por juicios relativos a actos genéricos” (Bayón, 1991, p. 321), de lo que surge el concepto de normas probabilísticas, para explicar las reglas que tienen un estándar débil o abierto. Sin embargo, solo se hacen formulaciones abiertas de manera descriptiva, pero estas no sirven como solución en el caso del conflicto entre deberes contenidos y las normas mencionadas. No obstante, estas son equiparadas por Bayón con los deberes prima facie, como herramienta para dirimir conflictos entre los mismos. Con todo,

es necesaria una determinación de la estructura de la deliberación moral, más exactamente en cuanto a sus puntos de partida (como principios o reglas morales), y su relación con los juicios morales particulares; con el fin de explicitar una estructura racional de la norma moral (Bayón, 1991, p. 322).

Por otra parte, siguiendo a Lyons, Bayón propone una distinción entre principios morales fuertes y principios morales débiles, atendiendo a "una representación esquemática de juicios o actos particulares frente a casos generales que determinen el tipo de regla moral en cuestión" (Bayón, 1991, p. 323). Dicha representación es: $(p \& q)$ entonces $r$, en donde la proposición $q$ determina el elemento contendor de una circunstancia fáctica, de un principio genérico determinado. En esta medida,

la formalización, como herramienta per sé, solo permite destacar una categorización de una norma moral a partir de un hecho fáctico genérico que le de origen, hasta tener como finalidad llegar a una construcción axiomática de formulaciones morales al modo de un principio débil (Bayón, 1991, p. 324).

Al final, son estas normas de corte hipotético las que abarcan, en forma más profunda, la genericidad propia de los principios que se enuncian en forma de reglas morales que, para el caso de este capítulo, son las normas constitutivas de derechos humanos. Es importante tener presente que dicha genericidad no puede constituir nunca una regla última de conducta, ni tampoco una estructura rígida de interpretación. En cambio, conforma 
modelos que permiten una gradación más o menos flexible de características morales que pueden adecuarse a preceptos normativos que regulan la aplicabilidad o no de principios extra normativos a casos concretos, es decir, a su adecuación al sistema del Derecho (Luzzatti, 2013, p. 134).

Esta fundamentación es posible por la interpretación de un principio moral, a partir del precepto fáctico que permite su axiomatización hacia arriba del ordenamiento jurídico, tanto nacional como supranacional.

\section{Conclusiones}

El debate sobre la inclusión de principios y valores contenidos en las reglas morales trasciende más allá de las categorías positivistas clásicas, ya que es un juicio en virtud del cual los criterios morales no pueden ser englobados en el estudio del Derecho.

El juicio basado en la inclusión contingente de los derechos humanos también presenta peculiaridades que afectan la estructuración lógica del sistema jurídico, porque su adecuación al ordenamiento se logra ajustando los principios y preceptos lógicos del mismo hacia su concreción, su garantía de cumplimiento y su estructuración en el marco del modelo positivista.

\section{Referencias}

Agudelo, O. (2017). Lógica tolerante, tercero incluido y permiso débil. En O. Agudelo, J. León, M. Prieto \& Y. Reyes, Lógica aplicada al razonamiento del derecho (pp. 149-152). Bogotá: Universidad Católica de Colombia.

Alchourrón, C. \& Bulygin, E. (2013). Introducción a la metodología de las ciencias jurídicas. Buenos Aires: Astrea S.A.

Ávila, H. (2011). Teoría de los principios. Madrid: Marcial Pons S.A.

Bayón, J. (1991). La normatividad del derecho: deber jurídico y razones para la acción. Madrid: Centro de Estudios Políticos y Constitucionales.

Bobbio, N. (1993). Liberalismo y democracia. Bogotá: Fondo de Cultura Económica.

Pico della Mirandola, G. (2010). Discurso sobre la dignidad del hombre. Revista digital universitaria, 11, 1-6.

Dworkin, R. (2012). Los derechos en serio. Barcelona: Ariel S.A. 
•Calificación lógico-moral de los derechos humanos.

Echave, D., Urquijo, M. \& Guibourg, R. (2008). Lógica, proposición y norma. Buenos Aires: Astrea S.A.

Fuller, L. (1967). La moral del derecho. México D.F.: Editorial Trillas S.A.

Garzón, E. (1997). Derecho y moral. En E. Garzón \& F. Laporta (Eds.), El derecho y la justicia (pp. 397-424). Madrid: Trotta S.A.

Guibourg, R. (2010). Derecho, sistema y realidad. Buenos Aires: Astrea S.A.

Haberle, P. (2003). La libertad fundamental en el Estado constitucional. Granada: Comares S.A.

Kelsen, H. (2009). El Estado como integración: una controversia de principio. Madrid: Tecnos S.A.

Luzzatti, C. (2013). El principio de autoridad y la autoridad de los principios. Madrid: Marcial Pons S.A.

Nino, C. S. (1989). Ética y derechos humanos. Buenos Aires: Astrea S.A.

Pérez Bermejo, J. (2010). Coherencia y sistema jurídico. Madrid: Marcial Pons S.A.

Von Wright, G. H. (1979). Norma y acción. Madrid: Tecnos S.A.

Von Wright, G. H. (1997). Normas, verdad y lógica. México D.F.: Fontamara S.A.

Von Wright, G. H. (2002). Sobre la libertad humana. Barcelona: Paidós S.A 
